\title{
REVISÃO INTEGRATIVA DE LITERATURA FATORES DE RISCO PARA O AGRAVAMENTO DA COVID-19 EM INDIVÍDUOS JOVENS
}

Viviane Vanessa Rodrigues da Silva Santana ${ }^{1}$

Keila Cristina Pereira do Nascimento Oliveira ${ }^{1}$

Katiane da Silva Mendonça ${ }^{1}$

Daise Tavares da Silva ${ }^{31}$

Hallana Laisa de Lima Dantas ${ }^{1}$

\author{
https://orcid.org/0000-0001-6903-1444 \\ https://orcid.org/0000-0003-0167-5889 \\ https://orcid.org/0000-0003-3740-9670 \\ https://orcid.org/0000-0003-3807-4294 \\ https://orcid.org/0000-0001-6721-0860
}

Objetivo: Descrever as evidências científicas que abordem os fatores de riscos associados à infecção por COVID-19 em individuos adultos jovens. Método: Trata-se de uma revisão integrativa da literatura em seis etapas, com uso do método PICO e Preferred Reporting Items for Systematic Reviews and Meta-Analyses. Utilizou-se os niveis de evidência de Oxford para classificação dos artigos. Resultados: Dos 42 artigos encontrados, sete atenderam aos critérios de inclusão e exclusão. Todos os estudos são publicações de 2020. Observou-se que as doenças crônicas são apresentadas como principais fatores de risco para o COVID-19, apenas dois artigos apresentaram características relacionadas aos casos de mortes e apontaram condições subjacentes como agravantes para o novo coronavírus. Os estudos também apontaram a população jovem como a mais acometida pela doença associado a apresentação de sintomas leves como tosse, febre e fadiga, com baixa taxa de mortalidade em comparação ao grupo de risco. Conclusão: Dentre os fatores de risco mais evidentes neste estudo pode-se ressaltar doenças cardiovasculares como a hipertensão, diabetes e tabagismo, apontando também uma maior prevalência de casos em indivíduos de sexo masculino.

Descritores: Adulto Jovem; Coronavirus; Fatores de Risco; Morbidade.

Risk factors for the worsening of covid-19 in young individuals

Objective: To describe the scientific evidence that addresses the risk factors associated with COVID-19 infection in young adult individuals. Method: This is an integrative review of the literature in six steps, using the PICO method and Preferred Reporting Items for Systematic Reviews and Meta-Analyzes. The Oxford levels of evidence were used to classify articles. Results: Of the 42 articles found, seven met the inclusion and exclusion criteria. All studies are publications from 2020. It was observed that chronic diseases are presented as the main risk factors for COVID-19, only two articles presented characteristics related to cases of death and pointed out underlying conditions as aggravating for the new coronavirus. The studies also pointed out the young population as the most affected by the disease associated with the presentation of mild symptoms such as cough, fever and fatigue, with a low mortality rate compared to the risk group. Conclusion: Among the most evident risk factors in this study, cardiovascular diseases such as hypertension, diabetes and smoking can be highlighted, also pointing to a higher prevalence of cases in male individuals.

Descriptors: Young Adult; Coronavirus; Risk Factors; Morbidity.

\section{Factores de riesgo para el empeoramiento de covid-19 en individuos jóvenes}

Objetivo: Describir la evidencia científica que aborda los factores de riesgo asociados con la infección por COVID-19 en individuos adultos jóvenes. Método: Esta es una revisión integradora de la literatura en seis pasos, utilizando el método PICO y los elementos de informes preferidos para revisiones sistemáticas y metaanálisis. Los niveles de evidencia de Oxford se utilizaron para clasificar los artículos. Resultados: De los 42 artículos encontrados, siete cumplieron los criterios de inclusión y exclusión. Todos los estudios son publicaciones de 2020. Se observó que las enfermedades crónicas se presentan como los principales factores de riesgo para COVID-19, solo dos artículos presentaron características relacionadas con casos de muerte y señalaron condiciones subyacentes como agravantes para el nuevo coronavirus. Los estudios también señalaron a la población joven como la más afectada por la enfermedad asociada con la presentación de sintomas leves como tos, fiebre y fatiga, con una baja tasa de mortalidad en comparación con el grupo de riesgo. Conclusión: Entre los factores de riesgo más evidentes en este estudio, se pueden destacar las enfermedades cardiovasculares como la hipertensión, la diabetes y el tabaquismo, lo que también apunta a una mayor prevalencia de casos en hombres.

Descriptores: Adulto Joven; Coronavirus; Factores de Riesgo; Morbilidad. 


\section{INTRODUÇÃO}

Detectada pela primeira vez em dezembro de $2019 \mathrm{em}$ Wuhan, China, a COVID-19 é uma doença respiratória emergente causada por um novo coronavírus, altamente infeccioso cujo seus principais sintomas clínicos incluem febre, tosse seca, fadiga, mialgia e dispnéia ${ }^{1}$. Os primeiros casos descritos foram associados a um mercado de frutos de mar e de animais silvestres vivos como morcego ${ }^{2}$.

Devido às proporções tomadas pela contaminação, a Organização Mundial da Saúde (OMS) declarou como pandêmico o surto de COVID-19, em 11 de março de $2020^{3}$. A alta prevalência dos coronavírus, e a ampla distribuição no mundo, está associado à sua diversidade genética e recombinação genética, em locais com intensa interação entre o homem e animais, facilita o surgimento periódico de novas cepas de coronavirus $^{2}$

Classificada como uma Zoonose, incluindo a mesma família dos Coronaviridae que ocorreram nas últimas décadas tais como, a síndrome respiratória aguda grave (SARS-CoV), e a Síndrome Respiratória do Oriente Médio (MERS-CoV), durante o surto atual o SARS-CoV-2 inicialmente chamado de novo coronavirus humano, é responsável pela doença denominada COVID-19 apresentando alta taxa de disseminação entre humanos $^{4}$.

O SARS-CoV-2 é o sétimo membro dos coronavirus que infecta humanos, acomete as células epiteliais alveolares pulmonares usando endocitose mediada por receptor através da enzima conversora de angiotensina II como receptor de entrada ${ }^{5}$. A transmissão mais provável ocorre por contato e gotículas respiratórias, e também por fômites contaminados por secreções respiratórias, o contato prolongado com casos de COVID-19 apresenta maior risco de contágio, a mesma também pode ocorrer a partir de pacientes assintomáticos ${ }^{6}$.

Independentemente da idade, todos os individuos, podem ser infectados pelo SARS-CoV-2 e apresentar a sindrome respiratória aguda. Sobretudo pessoas de meia-idade e idosos são os mais afetados por esta síndrome, que junto àqueles com comorbidades e imunossupressão mostraram-se particularmente propensos a quadros mais graves e necessidade de internamento em unidades de terapia intensiva. .

Foram confirmados no mundo 1.844 .863 casos de COVID-19 (71.779 novos em relação ao dia anterior) e 117.021 mortes (5.369 novas em relação ao dia anterior), até 14 de abril de 2020, pela Associação Pan-Americana de Saúde (OPAS). Esses índices indicam que mesmo diante de medidas protetivas como isolamento social, em todo os países afetados, os casos de notificados continuam crescente ${ }^{7}$.

Diante desse crescente aumento de morbidade, princi- palmente na população jovem em todo o mundo, este estudo justifica-se através da análise e identificação dos fatores de risco associados para o agravo da COVID-19 em adultos jovens, atraindo mais atenção para esse tema e corroborando com novos estudos que irão contribuir para promoção da saúde dessas pessoas. Visto isso, o presente estudo tem por objetivo descrever as evidências cientificas que abordem os fatores de riscos associados à infecção por COVID-19 em indivíduos adultos jovens.

\section{MÉTODO}

Trata-se de uma revisão integrativa da literatura. Este método de pesquisa consiste na construção de uma análise ampla sobre um determinado tema ${ }^{8}$. Dentre os métodos de revisão, esse tipo de estudo permite a inclusão simultânea de pesquisa experimental e quase-experimental, e ainda a combinação de dados de literatura teórica e empírica, proporcionando dessa forma uma compreensão mais completa do tema de interesse?

Para tanto foi adotado um protocolo com seis etapas indicadas para construção da revisão integrativa da literatura: 1) seleção da pergunta de pesquisa; 2) definição dos critérios de inclusão de estudos e seleção de amostras; 3) representação dos estudos selecionados através de fichamentos, considerando os pontos principais e características comuns entre eles; 4) análise crítica dos achados, identificando diferenças e conflitos; 5) interpretação dos resultados e 6) reportar, de forma clara, a evidência encontrada através da síntese. ${ }^{10}$

Foi utilizado o método Preferred Reporting Items for Systematic Reviews and Meta-Analyses (PRISMA), ${ }^{11}$ que consiste em um checklist com 27 itens, a questão norteadora foi formulada de acordo com a estratégia PICO, ${ }^{12}$ acrônimo que reúne os elementos: Paciente, Intervenção, Comparação (que nesta investigação não se aplica), Outcomes (desfecho), culminando respectivamente nos termos: individuos jovens, fatores de risco, morbidade, COVID-19 respectivamente, objetivando encontrar estudos pertinentes à pesquisa em questão. Ficou estabelecida a seguinte questão norteadora: quais são os fatores de risco associados a agravo da covid-19 em individuos jovens? Em seguida, realizou-se uma busca na literatura científica durante o mês de abril de 2020, utilizando-se dos seguintes critérios de inclusão: textos completos com livre acesso a bases de dados nos idiomas português, espanhol ou inglês, de relevância para o tema, clareza metodológica. Utilizando estratégia de busca avançada auxiliada pelo operador booleano "AND" combinou-se os descritores extraídos da lista de Descritores em Ciências da Saúde (DeCS) "Fatores de Risco", “Morbidade", “Mortalidade", “Adultos", “Adulto Jovem", "and covid 19" and "adulto jovem", à palavra-chave, que devido 
à abrupta emergência ainda não consta dentre DeCS, “COVID-19". Medline, Science Direct, LILACS, SCOPUS, BDEnf no mês de abril de 2020 .

Foram excluídos relatos de caso ou experiência, artigo de opinião, trabalhos de teses, monografias, dissertações (literatura cinzenta), publicações anteriores a novembro de 2019, pesquisa aplicada a animais. Artigos em duplicata foram considerados apenas uma vez.

O instrumento de avaliação adotado foi o delineamento da investigação dos niveis de Oxford Centre for Evidence-based Medicine, nesse método, os artigos foram classificados em recomendações de nível A, B, C e D e de acordo com nível de eficiência (la, lb, 1c, 2a, 2b, 2c, 3a, 3b, 4 e 5) ${ }^{13}$ (Quadro 1).Do material obtido, a fim de organizar e sumarizar os dados, os pesquisadores elaboraram um instrumento de agrupamento de dados, discriminando: título, país de estudo, categoria de estudo, natureza de estudo, método de análise, enfoque.

Quadro 1 - Níveis de Evidência Científica segundo a Classificação de Oxford Centre for Evidence-Based Medicine.

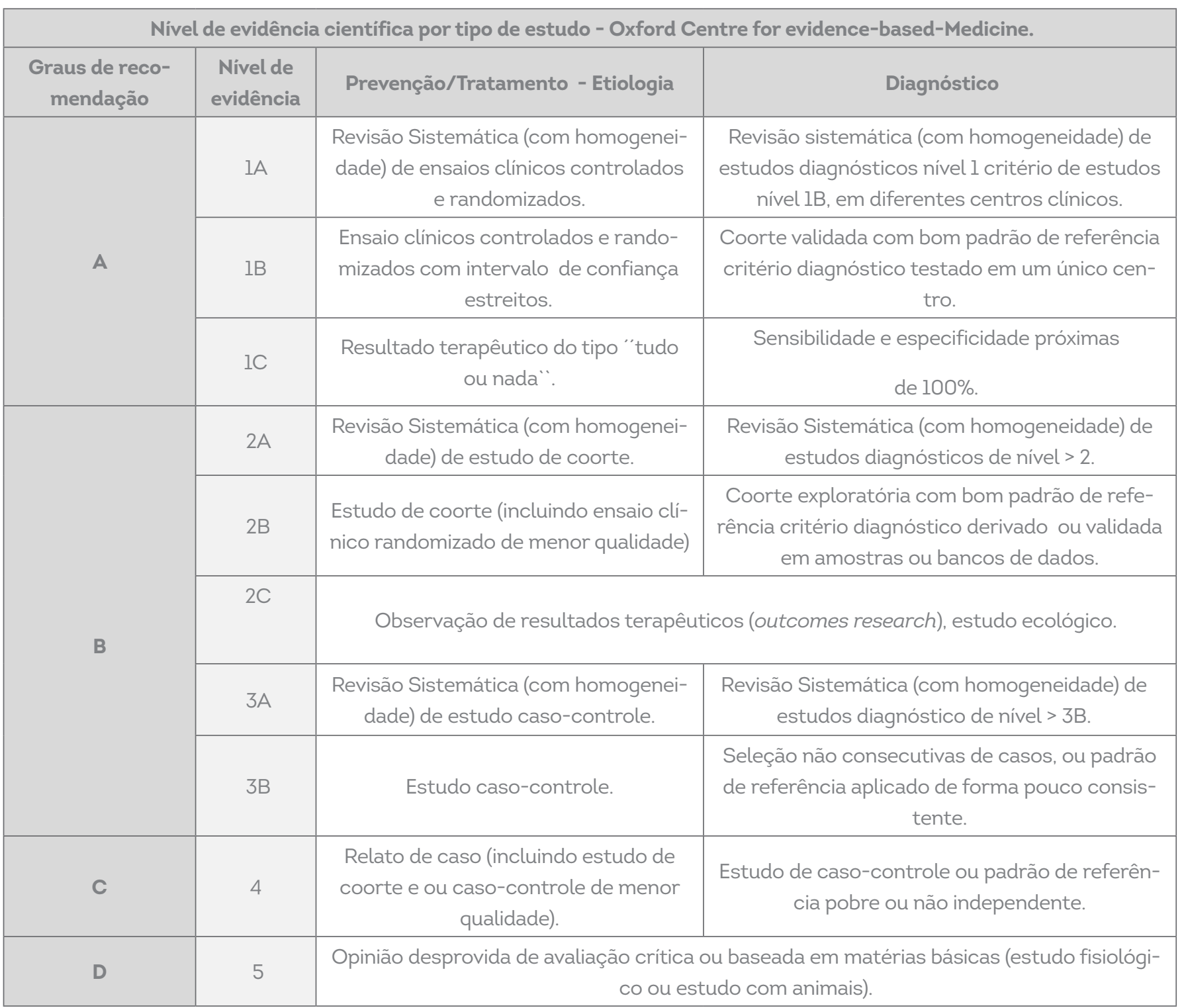

Fonte: Oxford Centre for Evidence-based Medicine: levels of evidence, $2009^{13}$.

Os apontamentos dos elementos essenciais para os fatores de riscos no agravamento da COVID-19 em indivíduos jovens foram apresentados por meio do agrupamento dos artigos em três categorias temáticas: taxa de morbidade e mortalidade e fatores associados à infecção da COVID-19 na população jovem. Os dados de cada artigo selecionado foram avaliados, discutidos e comparados sob o referencial teórico atual e pertinente para estudo, para subsidiar uma compreensão aprofundada e crítica do assunto. 
Foram selecionados 42 artigos, removidos os duplicados, restaram 34. Após a leitura prévia dos títulos foram selecionados 15 artigos. Com a aplicação dos critérios de inclusão e exclusão 1 artigo foi eliminado pois a faixa etá- ria não correspondia aos objetivos da pesquisa, portanto, foram selecionados 7 artigos (Fluxograma 1). Não foram encontrados trabalhos no banco de dados do LILACS e BDENF no momento desta pesquisa.

Fluxograma 1 - Fluxograma Prisma de Identificação, seleção e inclusão das publicações na amostra da revisão integrativa. Maceió, AL. Brasil, 2020.

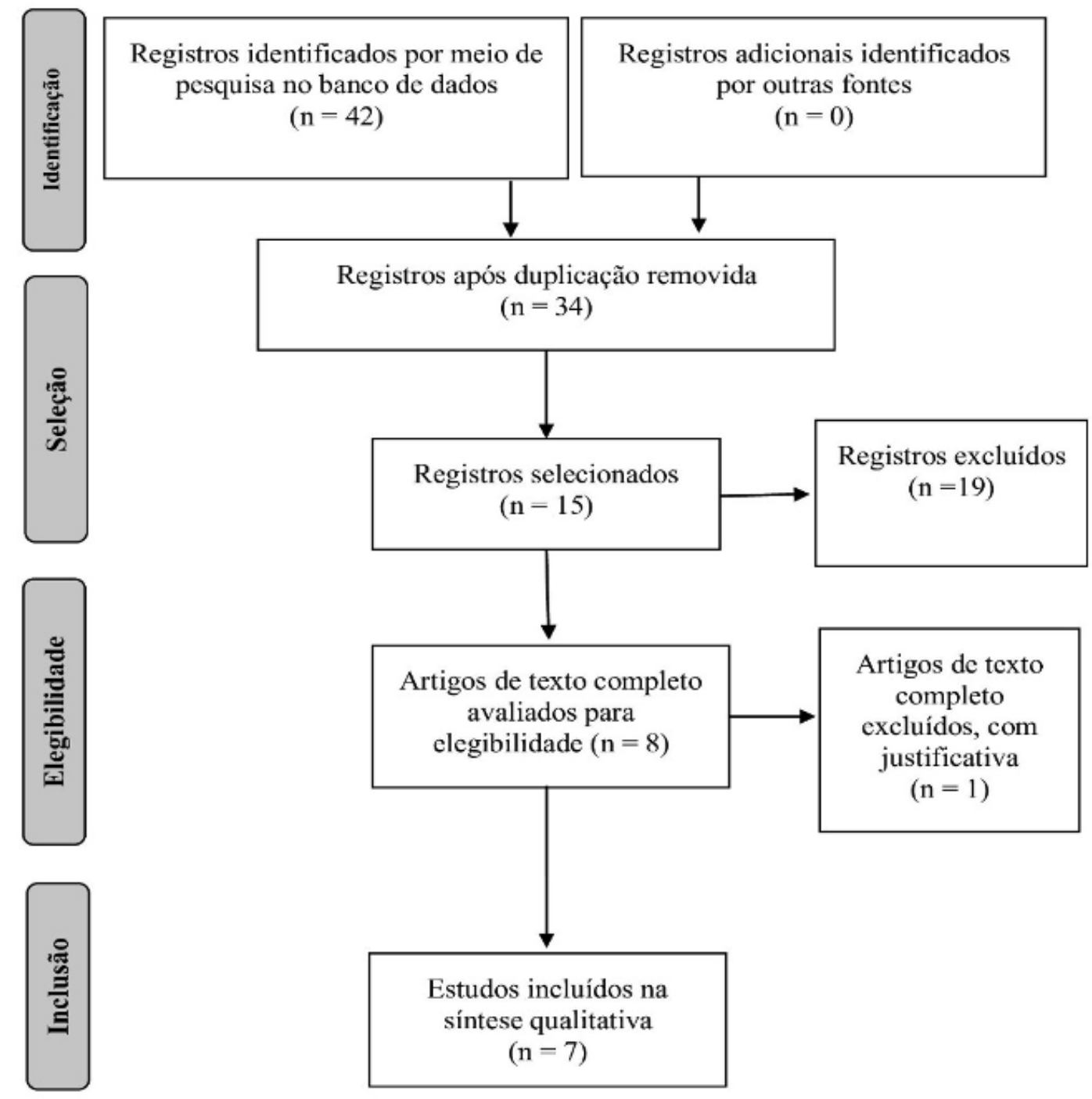

Fonte: Dados da pesquisa, 2020.

Posteriormente, procedeu-se a análise sistemática para caracterização dos estudo selecionados. Os trabalhos foram comparados e agrupados por similaridade e relevância de conteúdo para o trabalho. A revisão integrativa permitiu a categorização das evidências encontradas, alicerçadas em métodos avaliativos de rigor científico conceituando os pontos pertinentes ao estudo, havendo ainda a possibilidade de identificação de outras questões que possam compor investigações futuras.

A metodologia aplicada a esta investigação não requer aprovação do Comitê de Ética e Pesquisa. Por fim, declara-se para os devidos fins que não houve conflito de interesses.

\section{RESULTADOS}

Os sete artigos incluídos nesta revisão foram publicados em 2020, sendo um artigo publicado em fevereiro, cinco em março e um em abril. Quanto à origem, cinco foram realizados na China, um na Europa, e um na Coreia. As características dos estudos podem ser observadas no Quadro 2. 
Quadro 2 - Panorama das características dos estudos selecionados para identificação dos fatores de risco associados a agravos da COVID-19 em adultos jovens. Maceió-AL, Brasil, 2020.

\begin{tabular}{|c|c|c|c|c|}
\hline Id & Título & Local/Ano & $\begin{array}{c}\text { Tipo de estudo/nivel de } \\
\text { evidência }\end{array}$ & Achados principais \\
\hline El & $\begin{array}{l}\text { First cases of coronavirus } \\
\text { disease } 2019 \text { (COVID-19) } \\
\text { in the WHO European } \\
\text { Region, } 24 \text { January to } 21 \\
\text { February } 2020^{14}\end{array}$ & $\begin{array}{l}\text { Europa } \\
\text { Março/ } \\
2020\end{array}$ & $\begin{array}{l}\text { Estudo epidemiológico } \\
\text { descritivo/ 3A }\end{array}$ & $\begin{array}{l}\text { Foram notificados } 47 \text { casos. } \\
\text { Dos } 38 \text { casos estudados, } 21 \\
\text { estavam vinculados a dois } \\
\text { grupos na Alemanha e na } \\
\text { França, } 14 \text { estavam infecta- } \\
\text { dos na China. A idade média } \\
\text { dos casos foi de } 42 \text { anos; } 25 \\
\text { eram do sexo masculino. }\end{array}$ \\
\hline E2 & $\begin{array}{l}\text { Analysis on } 54 \text { Mortality } \\
\text { Cases of Coronavirus Dis- } \\
\text { ease } 2019 \text { in the Republic of } \\
\text { Korea from January } 19 \text { to } \\
\text { March 10, } 2020^{15}\end{array}$ & $\begin{array}{l}\text { CORÉIA } \\
\text { Março/ } \\
2020\end{array}$ & $\begin{array}{l}\text { Estudo epidemiológico } \\
\text { descritivo/ 2B }\end{array}$ & $\begin{array}{c}\text { O número global de casos } \\
\text { confirmados em } 15 \text { de mar- } \\
\text { ço de } 2020 \text { é de } 156.400 \text {, } \\
\text { com morte total em } 5.833 \\
\text { (3,7\%) em todo o mundo. A } \\
\text { taxa geral de casos fatais } \\
\text { de COVID-19 na Coréia foi } \\
\text { de } 0,7 \% \text { Sendo } 0,4 \% \text { entre } \\
\text { adultos jovens. }\end{array}$ \\
\hline E3 & $\begin{array}{l}\text { COVID-19: what has been } \\
\text { learned and to be learned } \\
\text { about the novel coronavirus } \\
\text { disease }^{16}\end{array}$ & $\begin{array}{l}\text { China } \\
\text { Março/ } \\
2020\end{array}$ & Estudo Descritivo/ 2A & $\begin{array}{l}\text { O COVID-19 tem menor } \\
\text { gravidade e mortalidade que } \\
\text { a SARS, mas são mais trans- } \\
\text { missivo e afeta mais indivi- } \\
\text { duos tanto jovens quanto } \\
\text { idosos e mais homens do } \\
\text { que mulheres. Ejá matou } \\
\text { mais de } 3.000 \text { pessoas e } \\
\text { infectou mais de } 80.000 \text { na } \\
\text { China. }\end{array}$ \\
\hline E4 & $\begin{array}{l}\text { Characteristics of COVID-19 } \\
\text { infection in Beijing }{ }^{17}\end{array}$ & $\begin{array}{l}\text { Cidade de } \\
\text { Pequim na China } \\
\text { Abril/ } \\
2020\end{array}$ & Estudo Retrospectivo/ 2B & $\begin{array}{l}\text { As características dos } \\
\text { pacientes infectados com } \\
\text { COVID-19 em Pequim eram } \\
\text { obviamente diferentes de } \\
\text { Wuhan, com menor fatalida- } \\
\text { de e maior taxa de alta. }\end{array}$ \\
\hline E5 & $\begin{array}{c}\text { Clinical course and risk } \\
\text { factors for mortality of adult } \\
\text { inpatients with COVID-19 in } \\
\text { Wuhan, China: a retrospec- } \\
\text { tive cohort study }{ }^{18}\end{array}$ & $\begin{array}{l}\text { China, cidade de Wuhan } \\
\qquad \begin{array}{c}\text { Março/ } \\
2020\end{array}\end{array}$ & $\begin{array}{l}\text { Estudo de coorte retrospec- } \\
\text { tivo e multicêntrico/ 2B }\end{array}$ & $\begin{array}{l}\text { A idade, maior escore SOFA } \\
\text { e d-dimero elevado na } \\
\text { admissão foram fatores de } \\
\text { risco para morte de pacien- } \\
\text { tes adultos com COVID-19 }\end{array}$ \\
\hline E6 & $\begin{array}{l}\text { Clinical characteristics of } \\
113 \text { deceased patients with } \\
\text { coronavirus disease 2019: } \\
\text { retrospective study. }{ }^{19}\end{array}$ & $\begin{array}{l}\text { Cidade de Wuhan, China } \\
\qquad \begin{array}{c}\text { Março/ } \\
2020\end{array}\end{array}$ & Estudo de Coorte/1B & $\begin{array}{l}\text { A infecção por COVID-19 } \\
\text { pode causar inflamação } \\
\text { pulmonar e sistêmica, dis- } \\
\text { função de múltiplos órgãos, } \\
\text { SDRA e insuficiência res- } \\
\text { piratória, sepse, lesão car- } \\
\text { diaca aguda e insuficiência } \\
\text { cardíaca foram às complica- } \\
\text { ções críticas. }\end{array}$ \\
\hline E7 & $\begin{array}{l}\text { Host susceptibility to severe } \\
\text { COVID-19 and establish- } \\
\text { ment of a host risk score: } \\
\text { findings of } 487 \text { cases out- } \\
\text { side Wuhan }{ }^{20}\end{array}$ & $\begin{array}{l}\text { China } \\
\text { Março/ } \\
2020\end{array}$ & $\begin{array}{l}\text { Estudo de Coorte retros- } \\
\text { pectivo/3A }\end{array}$ & $\begin{array}{l}\text { Os fatores de risco associa- } \\
\text { dos ao COVID-19 grave são } \\
\text { os mecanismos subjacentes } \\
\text { à progressão da doença. O } \\
\text { principal achado é de que a } \\
\text { hipertensão é um fator de } \\
\text { risco para COVID-19. }\end{array}$ \\
\hline
\end{tabular}


Quanto à abordagem, os sete estudos selecionados eram quantitativos, sendo $42,8(n=3)$ estudos epidemiológicos descritivos, $28,5 \%(n=3)$ estudo de coorte retrospectivo e $14,2 \%(n=1)$ estudo prospectivo, como observados no quadro 3. Quanto ao nível de evidência, os artigos obtidos foram classificados em 2 A (1 artigo), 2B (3 artigos), seguido de 3A (2 artigos) e IB (1 artigos). para entender sobre esse nível de evidência a explicação é encontrada no quadro 1 destacada na metodologia deste artigo.

Cinco estudos apresentaram a média da idade dos casos estudados, sendo a mais baixa de 38 anos e a mais alta de 56 anos, evidenciando uma incidência maior da infecção em pessoas fora da classe de risco, demonstrando que os adultos jovens foram mais acometidos pela COVID-19, porém com quadro mais leve da doença, inclusive um dos estudos analisados mostrou que $77,4 \%$ dos infectado eram adultos jovens. Notou-se também uma similaridade nos achados com relação a incidência da doença em pessoas do sexo masculino. Todos os sete artigos apresentaram maior número de casos em homens, no entanto ressaltaram que não há diferença significativa na média de idade entre homens e mulheres acometidos pelo novo coronavírus. ${ }^{14,15,16,17,18,19,20}$.

Dentre os estudos que abordam os fatores de risco todos apontam as comorbidades como fatores agravantes, dos quais seis pesquisas apresentaram doenças crônicas como insuficiência cardíaca e doenças cardiovasculares. Indivíduos com comorbidades cardiovasculares são mais propensos a desenvolver complicações cardíacas quando infectados pelo novo coronavírus 14,15,16,17,18,19,; quatro artigos citam a hipertensão como principal achado em relação aos fatores de riscos associados 14,18.19.20; um estudo apresentou a necessidade de suporte respiratório e a diabetes como agravante para infecção, pois segundo as evidências apontadas a chance de morte é maior nesse tipo de paciente ${ }^{20}$. Além disso, enfatiza que o tabagismo, e a obesidade também podem estar associados aos fatores de risco, e uma pesquisa mostrou que os fatores agravantes nos casos estudados foram idade avançada, lipopenia, leucocitose e ALT elevado, lactato desidrogenase, troponina I cardíaca de alta sensibilidade. ${ }^{18}$

Frente aos estudos analisados, não houve relatos de mortes em Adultos jovens. Esses indivíduos quando acometidos pela COVID-19 apresentam sintomas leves, sendo estes agravados quando associados a comorbidades subjacentes como as doenças crônicas.

\section{DISCUSSÃO}

Um estudo com 38 óbito na Europa apontou que a idade média dos casos de infecção ocorreu na faixa etária de
42 anos, variando de 2 a 81 anos. Dados apontam que sete desses indivíduos possuíam condições pré-existentes, sendo um obeso e um com doença cardiaca pré-existente. Não foram relatados dados sobre sinais clínicos, como dispnéia, para nenhum dos 38 casos. Dos 38 óbitos, 24 aconteceram com indivíduos com idade entre 18 e 49 anos, o que evidencia uma taxa de morbidade alta entre individuos jovens, mas com baixa letalidade. ${ }^{14}$ A mortalidade é a questão mais importante ao lidar com o surto inesperado ou esperado e ao estabelecer prioridades para controlar as epidemia. ${ }^{14-15}$

Há evidências em um estudo multicêntrico com 8.866 pacientes, incluindo 4.021 pacientes com COVID-19 confirmado, onde o vírus infectou pessoas de todas as idade. mas principalmente com 30 a 60 anos de idade, apresentou que os três fatores de risco principais para COVID-19 foram sexo (masculino), idade avançada (> 60 anos) e pneumonia grave ${ }^{16}$. Os pacientes que necessitam de terapia intensiva apresentam probabilidade maior de apresentar comorbidade e complicações subjacentes. Principalmente se levar em consideração a idade que conta como fator prognóstico para o resultado da COVID-19. O estudo ressalta a idade avançada, mas não desconsidera que se os fatores de riscos também se aplicam a indivíduos mais jovens. ${ }^{16-17}$

Em um recorte de 56 pacientes que foram admitidos em uma UTI, apenas 18 eram idosos e 38 jovens e de meia idade. ${ }^{21}$

Um estudo realizado na china abordou características clínicas de 113 pacientes, realizando associações entre pacientes graves com COVID-19 e as comorbidades crônicas, em seu relato aponta que a idade média dos pacientes falecidos foi de 68 anos (dentro de intervalo interquartil 62 a 77 anos), enquanto os pacientes recuperados apresentaram idade média de 51 anos (dentro do intervalo de 37 a 66 anos). Entre $63 \%$ dos pacientes que morreram e $39 \%$ que se recuperaram, apresentavam pelo menos uma condição médica crônica, ressalta-se que a hipertensão, diabetes, doença cardiovascular, doença cerebrovascular, ou doença pulmonar crônica são as comorbidades crônicas mais frequentes entre os pacientes falecidos. A obesidade e tabagismo também foram associados a riscos aumentados para comorbidade da COVID-19.19-20

Pesquisas apontam que ter um histórico de tabagismo pode aumentar significativamente as chances de apresentar resultados adversos à saúde de pacientes acometidos pela COVID-19, favorecendo a necessidade de internação em terapia intensiva e ventilação mecânica, sendo esse classificado como um fator de risco para muitas outras infecções respiratórias, além de estar associado com aumento do desenvolvimento da sindrome do desconforto respiratório agudo, uma complicação importante para casos graves da 


\section{COVID-19.22}

O tabaco pode ser consumido de diversas maneiras como no narguilé, muitos fumantes praticam esse ato na companhia de amigos e familiares, sendo compartilhado pelo grupo, é uma prática bem conhecida e disseminada, especialmente entre os jovens, portanto, seu uso além de ser um fator de risco para doenças respiratórias devido ao acometimento fisiopatológico, atua também como um fômite para o SARS-CoV-2, sua má higienização pode fornecer um ambiente que promove a sobrevivência de microrganismos, esse procedimento de limpeza deve ser minucioso demandando tempo e trabalho, e deveria ocorrer a cada sessão de fumo. ${ }^{23}$

Nesse contexto, a educação em saúde mostra-se um forte aliado, como instrumento de promoção de informação para evitar o contágio, sendo esta uma das atribuições imprescindiveis do enfermeiro no processo de cuidar da saúde dos indivíduos. ${ }^{24}$ Essas informações podem ser propagadas através do ambiente tecnológico obtendo dessa forma um maior alcance, levando em conta que a profissão de enfermagem gera, manuseia, processa e comunica as informações de saúde com a equipe multiprofissional, os pacientes, familiares e a comunidade. ${ }^{25}$

Em uma pesquisa recente que avaliou os fatores de riscos para morte hospitalar pelo novo coronavírus, detectou-se que $48 \%$ dos pacientes apresentavam comorbidades, sendo a hipertensão a mais comum (30\%) seguido de Diabetes (19\%) e doença Coronariana (8\%). A média de idade da pesquisa foi de 56 anos variando de 18 a 87 anos. ${ }^{18} \mathrm{O}$ mesmo cita como fatores agravantes para mortes de adultos os níveis de dimero- $d$ superiores a $1 \mathrm{mg} / \mathrm{ml}$, níveis de $\mathrm{IL}-6$ mais elevados no sangue, troponina I cardíaca de alta sensibilidade e desidrogenase e lactopenia de lactato mais comum em casos graves da COVID-19.18

Outros autores apontam que há uma prevalência aparentemente alta de hipertensão arterial sistêmica em casos graves da COVID-19 sendo assim um fator de risco, isso devido ao comportamento do SARS-CoV-2, que pode usar a enzima conversora de angiotensina II, potencialmente regulada por bloqueadores do sistema renina-angiotensina utilizados como classe medicamentosa dá para essa comorbidade crônica, como um receptor de entrada viral nas células pulmonares. ${ }^{26}$

A infecção por SARS-CoV-2 em pacientes com diabetes em especial diabetes tipo 2 possivelmente desencadeia condições de estresse mais altas, causando uma resposta inflamatória e imunológica que pode manifestar a hiper ou hipoglicemia o que acaba interferindo na virulência da SARS-CoV-2. ${ }^{27}$ A hipoglicemia por exemplo mobiliza monócitos pró-inflamatórios e aumenta a reatividade plaquetária, con- tribuindo para uma maior mortalidade cardiovascular em pacientes com diabetes. ${ }^{28} \mathrm{~A}$ hiperglicemia está associado à resposta proliferativa de linfócitos inibida, ou seja, uma diminuição da resposta imune frente a diferentes tipos de estímulos, deixando o sistema imunológico menos eficiente. ${ }^{29}$

\section{Limitações do estudo}

Considerando a fase inicial da pandemia, este estudo apresentou como limitação o número reduzido de publicações que associam fatores de riscos para o agravo da COVID-19 com adultos jovens. Assim, pontua-se a importância da realização de novos estudos sobre a temática, especialmente no Brasil.

\section{Contribuições do Estudo para a Prática}

Almeja-se produzir literatura confiável sobre o tema e promover conhecimento científico sobre os fatores de risco associados ao agravamento da COVID-19 em indivíduos jovens. A fundamentação teórica, assim como, as análises das experiências e dos contextos dos fatores de risco para o agravamento da COVID-19 em adultos jovens, auxiliam na identificação de medidas assistenciais de saúde de forma mais assertivas, oferecendo suporte e orientação à conduta profissional de enfermagem. Este trabalho inicia e desperta investigações de aprofundamentos dentre a comunidade científica de enfermagem, de modo a fortalecer as medidas preventivas de contágio.

\section{CONCLUSÃO}

Os resultados deste estudo evidenciam que adultos jovens são comumente acometidos pela COVID-19 e podem evoluir com agravamento da doença quando apresentam fatores de risco como: doenças cardiovasculares como a hipertensão, diabetes, tabagismo e obesidade. A pesquisa aponta também uma maior prevalência de casos em indivíduos de sexo masculino. Contudo se faz necessários novos estudos para entender melhor os fatores de risco associado a indivíduos jovens acometidos pela COVID-19.

Contribuições dos Autores: Viviane Vanessa Rodrigues da Silva Santana: concepção e/ou desenho do estudo; redação e/ou revisão crítica do manuscrito; aprovação da versão final a ser publicada. Keila Cristina Pereira do Nascimento Oliveira: redação e/ou revisão crítica do manuscrito; aprovação da versão final a ser publicada. Katiane da Silva Mendonça: coleta, análise e interpretação dos dados: redação e/ou revisão crítica do manuscrito. Daíse Tavares da Silva: coleta, análise e interpretação dos dados; redação e/ou revisão crítica do manuscrito. Hallana Laisa de Lima Dantas: redação e/ou revisão crítica do manuscrito e aprovação da versão final a ser publicada. 


\section{REFERÊNCIAS}

1. Zhong BL, Luo W, Li HM, Zhang QQ, Liu XG, Li WT, Li Y. Knowledge, attitudes, and practices towards COVID-19 among Chinese residents during the rapid rise period of the COVID-19 outbreak: a quick online cross-sectional survey. Int J Biol Sci [Internet]. 2020 [cited 2020 apr 12]; 16(10): 1745-1752. Available from:http://www.ijbs.com/ v16pl745.htm

2. Sáfadi MAP, Rodrigues C, Carvalho AP, Pimentel AM, Berezin EN, Coser E, et al. Novo coronavirus (COVID-19). Dep Científico Infectol • Soc Bras Pediatr [Internet]. 2020 [cited 2020 apr 12]; 14:1-12. Available from: https://www. sbp.com.br/fileadmin/user_upload/22340d-DocCientifico_-_Novo_coronavirus.pdf

3. Organização Mundial da Saúde (OMS). O surto de 2019 CoV é uma emergência de preocupação internacional [Internet]. 2020 [cited 2020 apr 15]. Available from: http:// www.euro.who.int/en/health-topics/emergencies/pages/ news/news/2020/01/2019-ncov-outbreak-is-an-emergency-of-international-concern

4. Gorbalenya AE, Baker SC, Baric RS, Groot RJ, Drosten C, Gulyaeva AA, et. al. Severe acute respiratory syndrome-related coronavirus: The species and its viruses - a statement of the Coronavirus Study Group. Nat Microbiol [Internet]. 2020 [cited 2020 apr 15]; (5): 536-544. Available from: https://www.biorxiv.org/content/10.1101/2020.02.07 .937862 vl.full

5. Valero-Cedeño NJ, Mina-Ortiz JB, Veliz-Castro TI, Merchán-Villafuerte KM, Perozo-Mena AJ. COVID-19: La nueva pandemia con muchas lecciones y nuevos retos. Revisión Narrativa. Kasmera [Internet]. 2020 [cited 2020 apr 16]; 48(1):e48102042020. Available from: https://zenodo.org/record/3745322\#.Xt-oxOVKjIV

6. Cuesta E. La pandemia por el nuevo coronavirus covid-19. Rev Fac Cienc Med [Internet]. 2020 [cited 2020 apr 16]; 77(1): 1-3. Available from:https://revistas.unc.edu. ar/index.php/med/article/view/27935/29224

7. Organização Pan-Americana de Saúde (OPAS). Folha informativa- COVID-19 (Doença causada pelo novo coronavirus) [Internet]. 2020[cited 2020 apr 16]. Available from:https://www.paho.org/bra/index.php?option=com_ content\&view=articlefid=6101:covidl9\&Itemid=875

8. Souza MT, Silva MD, Carvalho R. Revisão integrativa: o que é? Como fazer isso?. Einstein. [Internet]. 2010 [cited 2020 apr 17]; (8):102-106. Available from: https://www. scielo.br/pdf/eins/v8nl/pt_1679-4508-eins-8-1-0102. pdf

9. Ercole FF, Melo LS, Alcoforado CLGC. Revisão Integrativa versus Revisão Sistemática. Rev Min Enferm [Internet]. 2014 [cited 2020 apr 17]; 18(1): 1-260. Available from: https://pesquisa.bvsalud.org/enfermeria/resource/pt/ bde-25575

10. Mendes KDS, Silveira RCCP, Galvão CM. Revisão integrativa: método de pesquisa para a incorporação de evidências na saúde e na enfermagem. Texto Contexto Enferm [Internet]. 2008 [cited 2020 apr 17]; 17(4): 758-64. Available from: https:// www.scielo.br/scielo.php?script=sci_arttextEpi$d=$ do104-07072008000400018\&lng=ptEtlng=pt

11. Moher D, Liberati A, Tetzlaff J, Altman DG, The PRISMA Group (2009) Itens de relatório preferidos para revisões sistemáticas e metanálises: The PRISMA Statement. PLoS Med [Internet]. 2009 [cited 2020 apr 17]; 6(7):el000097. Available from: https://journals.plos.org/ plosmedicine/article?id=10.1371/journal.pmed.1000097

12. Santos CMC, Pimenta CAM, Nobre MRC. A estratégia PICO para a construção da pergunta de pesquisa e busca de evidências. Rev. Latino-Am Enfermagem [Internet]. 2007 [cited 2020 apr 17]; 15(3). DOI: https://doi. org/10.1590/S0104-11692007000300023

13. Oxford Centre for Evidence-based Medicine: levels of evidence [Internet]. 2009 Mar [cited 2020 apr 17]. Available from: http://www.cebm.net/oxford-centre-evidence-based-medicine-levels-evidence-march-2009

14. Spiteri G, Fielding J, Diercke M, Campese C, Enouf V, Gaymard A, et. al. First cases of coronavirus disease 2019 (COVID-19) in the WHO European Region, 24 January to 21 February 2020. Euro Surveill [Internet]. 2020 [cited 2020 apr 18]; 25(9):2000178. Available from: https://www.eurosurveillance.org/content/10.2807/1560-7917.ES.2020.25.9.2000178

15. Korean Society of Infectious Diseases and Korea Centers for Disease Control and Prevention. Analysis on 54 Mortality Cases of Coronavirus Disease 2019 in the Republic of Korea from January 19 to March 10, 2020. J Korean Med Sci [Internet]. 2020 [cited 2020 apr 
18] ;35(12):el32. Available from:https://jkms.org/DOIx. php?id=10.3346/jkms.2020.35.el32

16. Yi Ye, Lagniton PNP, Ye S, Li E, Xu RH. COVID-19: COVID-19: what has been learned and to be learned about the novel coronavirus disease. Int $\mathrm{J}$ Biol Sci [Internet]. 2020 [cited 2020 apr 18]; 16(10): 1753-1766. Available from: http://www.ijbs.com/ v16pl753.htm

17. Tian S, Hu N, Lou J, Chen K, Kang X, Xiang Z, et al. Characteristics of COVID-19 infection in Beijing. Jour of Infect [Internet]. 2020 [cited 2020 apr 18]; 1(80):401406. Available from: https://www.journalofinfection. com/article/S0163-4453(20)30101-8/pdf

18. Fei Z, Ting Y, Ronghui D, Guohui F, Ying L, Zhibo L, et. al. Clinical course and risk factors for mortality of adult inpatients with COVID-19 in Wuhan, China: a retrospective cohort study. Lancet [Internet].2020 [cited 2020 apr 20]; 395(10229): 1054-1062. Available from:https:// www.thelancet.com/pdfs/journals/lancet/PIISO1406736(20)30566-3.pdf

19. Tao C, Di W, Huilong C, Weiming Y, Danlei Y, Guang C, et al. Clinical characteristics of 113 deceased patients with coronavirus disease 2019: retrospective study. BMJ [Internet]. 2020 [cited 2020 apr 18]; 368:ml091. Available from: https://www.bmj.com/content/368/bmj. m1091

20. Shi Y, Yu X, Zhao H, Wang H, Zhao R, Shenf J. Host susceptibility to severe COVID-19 and establishment of a host risk score: findings of 487 cases outside Wuhan. Crit Care [Internet]. 2020 [cited 2020 apr 19]; 24(1):108. Available from:https://ccforum.biomedcentral.com/ track/pdf/10.1186/s13054-020-2833-7

21. Liu K,Chen Y, Lin R, Han K. Clinical feature of COVID-19 in elderly patients: A comparison with young and middle-aged patients. Journal of Infection [Internet]. 2020[cited 2020 apr 18]; 80(6):14 18. Available from: https://pubmed.ncbi.nlm.nih.gov/32171866/

22. Huang C, Wang Y, Li X, Ren L, Zhao J, Hu Y, et al. Clinical features of patients infected with 2019 novel coronavirus in Wuhan, China. Lancet [Internet]. 2020[cited 2020 apr 20]; 395:497-506. Available from:https://www.thelancet.com/action/showPdf?pii=S0140-6736\%2820\%2930183-5
23. Instituto Nacional de Câncer José Alencar Gomes da Silva (INCA). Uso de narguilé: efeitos sobre a saúde, necessidades de pesquisa e ações recomendadas para legisladores [Internet]. 2017 [cited 2020 apr 20]. 2. ed. 49 p. Available from:https://www.who.int/tobacco/publications/prod_regulation/waterpipesecondedition/en/

24. Farias DLS, Nery RNB, Santana ME. O enfermeiro como educador em saúde da pessoa estomizada com câncer colorretal. Enferm Foco [Internet]. 2019 [cited 2020 jun 07]; 10(1): 35-39. Available from: http:// revista.cofen.gov.br/index.php/enfermagem/article/ view/1486/490

25. Machado ME, Paz AA, Linch GFC. Uso das tecnologias de informação e comunicação em saúde pelos enfermeiros brasileiros. Enferm Foco [Internet]. 2019 [cited 2020 jun 07];10(5): 91-96. Available from: http:// revista.cofen.gov.br/index.php/enfermagem/article/ view/2543/639

26. Kreutz R, Algharably EAEH, Azizi M, Dobrowolski P, Guzik T, Januszewicz A, et al. Hypertension, the renin-angiotensin system, and 'the risk of lower respiratory tract infections and lung injury: implications for COVID-19. Cardiovasc Res [Internet]. 2020 [cited 2020 apr 21]; 1(1):1-12. Available from:https:// academic.oup.com/cardiovascres/advance-article/ doi/10.1093/cvr/cvaa097/5819836

27. Wang A, Zhao W, Xu Z, Gu J. Timely blood glucose management for the outbreak of 2019 novel coronavirus disease (COVID-19) is urgently needed. Diabetes Res Clin Pract [Internet]. 2020 [cited 2020 apr 22]; 162:108118. Available from: https://doi.org/10.1016/j. diabres.2020.108118

28. Hussain A, Bhowmik B, do Vale Moreira NC. COVID-19 and diabetes: Knowledge in progress. Diabetes Res Clin Pract [Internet]. 2020 [cited 2020 apr 22]; 162:108142. Available from: https://doi. org/10.1016/j.diabres.2020.108142

29. Iqbal A, Prince LR, Novodvorsky P, Bernjak A, Thomas MR, Birch L, et al. Effect of hypoglycemiaon inflammatory responsesand the response to lowdose endotoxemia in humans. J Clin Endocrinol Metab [Internet]. 2019 [cited 2020 apr 22]; 104(4):1187-99. Available from:https://doi.org/10.1210/jc.2018-01168 